Central Washington University

ScholarWorks@CWU

All Faculty Scholarship for the College of the Sciences

$2-27-2020$

\title{
Detection of neopterin in the urine of captive and wild platyrrhines
}

\author{
Alexandra J. Sacco \\ Washington University in St. Louis \\ Jessica A. Mayhew \\ Central Washington University \\ Mrinalini Watsa \\ University of Missouri-St. Louis \\ Gideon Erkenswick \\ Washington University School of Medicine \\ April K. Binder \\ Central Washington University
}

Follow this and additional works at: https://digitalcommons.cwu.edu/cotsfac

Part of the Animal Sciences Commons

\section{Recommended Citation}

Sacco, Alexandra J.; Mayhew, Jessica A.; Watsa, Mrinalini; Erkenswick, Gideon; and Binder, April K., "Detection of neopterin in the urine of captive and wild platyrrhines" (2020). All Faculty Scholarship for the College of the Sciences. 96.

https://digitalcommons.cwu.edu/cotsfac/96

This Article is brought to you for free and open access by the College of the Sciences at ScholarWorks@CWU. It has been accepted for inclusion in All Faculty Scholarship for the College of the Sciences by an authorized administrator of ScholarWorks@CWU. For more information, please contact scholarworks@cwu.edu. 


\title{
Detection of neopterin in the urine of captive and wild platyrrhines
}

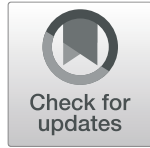

Alexandra J. Sacco ${ }^{1,2^{*}}$ D, Jessica A. Mayhew ${ }^{2,3}$, Mrinalini Watsa ${ }^{4,5,6}$, Gideon Erkenswick ${ }^{6,7}$ and April K. Binder ${ }^{2,8}$

\begin{abstract}
Background: Non-invasive biomarkers can facilitate health assessments in wild primate populations by reducing the need for direct access to animals. Neopterin is a biomarker that is a product of the cell-mediated immune response, with high levels being indicative of poor survival expectations in some cases. The measurement of urinary neopterin concentration (UNC) has been validated as a method for monitoring cell-mediated immune system activation in multiple catarrhine species, but to date there is no study testing its utility in the urine of platyrrhine species. In this study, we collected urine samples across three platyrrhine families including small captive populations of Leontopithecus rosalia and Pithecia pithecia, and larger wild populations of Leontocebus weddelli, Saguinus imperator, Alouatta seniculus, and Plecturocebus toppini, to evaluate a commercial enzyme-linked immunosorbent assay (ELISA) for the measurement of urinary neopterin in platyrrhines.

Results: Our results revealed measured UNC fell within the sensitivity range of the assay in all urine samples collected from captive and wild platyrrhine study species via commercial ELISA, and results from several dilutions met expectations. We found significant differences in the mean UNC across all study species. Most notably, we observed higher UNC in the wild population of L. weddelli which is known to have two filarial nematode infections compared to S. imperator, which only have one.

Conclusion: Our study confirms that neopterin is measurable via commercial ELISA in urine collected from captive and wild individuals of six genera of platyrrhines across three different families. These findings promote the future utility of UNC as a promising biomarker for field primatologists conducting research in Latin America to non-invasively evaluate cell-mediated immune system activation from urine.
\end{abstract}

Keywords: Neopterin, Health monitoring, Platyrrhines, Immune function, Biomarker

\section{Background}

Health and disease monitoring in wildlife has an important role in modern conservation efforts [1, 2]. Identifying health trends and evaluating disease prevalence and onset not only allows for the prevention of disease transmission to threatened populations, but also enhances our understanding of naturally occurring pathogen tolerance and susceptibility in wild populations. Evaluating the health status of wildlife allows researchers to anticipate the likelihood of disease outbreaks and infection as well as predict survival outcomes on an individual basis [3].

\footnotetext{
* Correspondence: Alexandra.Sacco@wustl.edu

${ }^{1}$ Department of Anthropology, Washington University in St. Louis, St. Louis, MO, USA

${ }^{2}$ Primate Behavior Program, Central Washington University, Ellensburg, WA, USA

Full list of author information is available at the end of the article
}

There are two general approaches to assessing wildlife health. The first focuses on evaluating physiological parameters associated with homeostasis and immune system activity, such as temperature, blood pressure, hematology or serum chemistry [4-13]. The second approach focuses on biomarkers indicative of illness and disease, which in some cases can be measured noninvasively. Commonly measured parameters that adaptively change in response to the disruption of homeostasis include cortisol measured in blood and feces [14-18], the gut microbiome evaluated in feces [19-25], and cpeptide measured in urine [26-29].

A little-explored but particularly promising biomarker for field biologists is neopterin, or $\mathrm{d}$ erythro-trihydroxypropylpterin, which is a molecule that is synthesized during the cell-mediated immune response [30]. Neopterin is a pteridine derivative, 
secreted by monocytes and macrophages upon the activation of cytokine interferon gamma (IFN- $\gamma$ ) [31]. The release of neopterin stimulates T-helper 1 (Th1) cell activation in immune responses to acute stress, viruses, or inflammation [31, 32]. In humans, elevated neopterin concentrations have been correlated with severe viral (e.g., cytomegalovirus infections, influenza) and parasitic infections (e.g., acute malaria) [31]. Neopterin concentrations have been used for diagnosis and prognosis in individuals with diseases that compromise immune responses, such as HIV [33]. In addition to disease response, neopterin is also associated with immunosenescence, or the agerelated decrease in cellular immune response [34-36]. Thus, neopterin is a reliable indicator of immune system activity in cases of compromised health status in humans.

Because the maintenance of a well-functioning immune system is critical to survival and resistance to infection based on human research [31], neopterin is a promising method of evaluating risk factors for disease transmission in threatened wildlife populations as well. Neopterin concentrations can be measured in serum, arterial blood, and urine using marker-specific immunoassays [31]. UNC is typically expressed as a ratio of neopterin to urinary creatinine $(\mathrm{Cr})$ to control for variation in urine density and concentration [31].

Urinary neopterin in particular is encouraging due to its high stability even under field conditions without refrigeration. Heistermann and Higham [37] found that urinary neopterin concentrations (UNC) can remain stable in urine stored at room temperature without preservatives for up to 21 days, however, urinary $\mathrm{Cr}$ is sensitive to degradation and has been shown to decrease over this same duration [37]. The degradation of Cr over time can be corrected for by using the specific gravity (SG) of the urine sample [38]. Short-term fecal and soil contamination do not significantly degrade UNC; however, UNC is sensitive to direct sunlight exposure and should be stored in a dark place [37]. These factors make UNC an ideal indicator for the immune status of wild nonhuman primate populations.

Urinary neopterin, which can be sampled with relative ease, has successfully been tested as an indicator of immune system activation in four non-human primate species over eight studies, five of which were in wild populations [3, 36, 37, 39-43]. Behringer et al. [39] found that two populations of wild chimpanzees (Pan troglodytes) had higher UNC than captive chimpanzees, suggesting that wild populations invest more energy into immune function with respect to captive non-human primates [39]. Elevated UNC was recorded in captive bonobos (Pan paniscus) with respiratory infections [40], captive SIV-infected rhesus macaques (Macaca mulatta)
[41, 42], and during a respiratory outbreak in a wild population of chimpanzees [3]. Remarkably, elevated UNC has also been observed in aging semi-free ranging Barbary macaques (Macaca sylvanus), suggesting that $\mathrm{UNC}$ may be an indicator of immunosenescence in nonhuman primates [36]. UNC was also correlated with serum neopterin and responsive to SIV-infection in captive rhesus macaques [42]. Although these studies have confirmed that UNC can be used to accurately represent and monitor changes in immune system activity in some primates, it can be impacted by seasonal fluctuations and temporal delays between the time of infection and immune response [43]. Lohrich et al. [43] found seasonal fluctuation in UNC of wild chimpanzees, with increased levels corresponding to low ambient temperature. Thus, $\mathrm{UNC}$ is a measure of monitoring immune function that requires frequent sampling over time to adequately detect changes in immune system activation during acute outbreaks [31, 43].

The use of neopterin as a non-invasive biomarker in platyrrhines is not well-represented in the literature. Lahoz et al. (2009) measured neopterin in the plasma of captive capuchins (Cebus apella) to assess the pharmacology of IFN $\beta$ and suitability of platyrrhines as an animal model for IFN $\beta$ research. They found that neopterin plasma concentrations increased several hours after subcutaneous injection of IFN $\beta$ 1a, a type 1 interferon that elicits immunomodulatory responses. Twentyfour hours after injection, a three-fold change in neopterin concentrations was observed, with a slow return to baseline levels after $48 \mathrm{~h}$. This study demonstrated that neopterin fluctuates in response to IFN $\beta$ 1a in this species, and that a commercially available ELISA kit allows for precise quantification of changes in neopterin plasma concentrations in C. apella [44]. This is the only study to measure neopterin plasma concentrations in platyrrhines, and no studies have measured UNC in platyrrhines thus far. The divergent evolution of platyrrhines is hypothesized to have contributed to the observed differences in the structure and protein-coding regions of other biomarkers, such as insulin and c-peptide [45], that have contributed to reduced biological potency and immunoreactivity of radioimmunoassays that were otherwise successful for measurements in catarrhines. Platyrrhines diverged from catarrhines $\sim 37 \mathrm{Ma}$ [46]; thus, it is advisable to measure UNC across multiple platyrrhine species. With 37 endangered primate species in the Neotropics, 15 of which are critically endangered [47], a stable biomarker of immune system activity could also allow for future health monitoring and accurate assessment of disease onset in platyrrhine populations. To address this need, we aimed to evaluate a commercially available neopterin ELISA for the detection of UNC across three platyrrhine families. To facilitate future 
comparisons to previous studies that have employed commercial neopterin kits [3, 36, 37, 39-44], we chose to use the same commercial ELISA kit used in these studies.

\section{Results}

Using a commercially available ELISA kit from IBL International GmbH (Art. No. RE59321, Hamburg, Germany), we successfully detected neopterin in urine samples opportunistically collected from two golden lion tamarins and two white-faced sakis living in captivity, and 40 emperor tamarins, 30 saddleback tamarins, one brown titi monkey, and one Colombian red howler monkey living in the wild.

We found no significant change in SG between the time of collection and the time of analysis in Pithecia pithecia (Wilcoxon signed-rank test, $\mathrm{W}=11, p=0.42$ ), Saguinus imperator (Wilcoxon signed-rank test, W = 1105, $p=0.71$ ), and Leontocebus weddelli (Wilcoxon signed-rank test, $\mathrm{W}=598.5, p=0.10$ ) (Table 1). The measured specific gravities for both samples collected from the Alouatta seniculus and Plecturocebus toppini individuals were the same at the time of collection and time of analysis (Table 1). The small number of samples collected from Leontopithecus rosalia $(n=2)$ precluded our ability to use statistical tests to evaluate the significance of SG changes, however, the observed difference was only 0.001 , a change that mirrors the nonsignificant SG changes in the other study species (Table 1 ). We measured lower SG values in the urine of wild species compared to captive species (Table 1); however, null hypothesis statistical tests were inappropriate in this instance due to potential species differences and the small sample size from captive species $(n=7)$. Therefore, we were unable to further explore the significance of these differences between wild and captive individuals.

All urine samples were read photometrically at $405 \mathrm{~nm}$ to measure UNC (Table 2). We demonstrated a significant difference in the UNC measured in $S$. imperator and $L$. weddelli $(t(50.68)=3.85, p<0.001)$, as urine samples collected from $L$. weddelli had a much higher measured UNC on average (Table 2).

High and low controls were measured within the manufacturer's acceptable range across three different wavelengths. Measured UNC decreased at expected intervals in accordance with each dilution factor (Fig. 1). The mean standard deviation of measured UNC in L. weddelli and S. imperator, which were sampled multiple times, was $38.50 \mathrm{ng} / \mathrm{mL}$ corr. SG, which is $3.9 \mathrm{x}$ and $7.8 \mathrm{x}$ lower than the observed inter-individual standard deviation across individuals of $S$. imperator and $L$. weddelli, respectively (Table 2 ). The intra-individual variation was further assessed using a paired $t$-test, which indicated that intra-individual variation was non-significant in these individuals $(t(5)=-0.92, p=0.40)$.

We suggest that the minimum threshold for biologically significant variation in immune function would be an effect size greater than chance (0.5). Considering measured UNC values in $L$. weddelli and S. imperator in our study, we calculated an effect size of 1.23. Calculations of sample size necessary to statistically differentiate observed levels of UNC ( $\beta=0.8, \alpha=.05)$, using formula $\left[d=\frac{m 1-m 2}{\sigma}\right]$, suggests a minimum sample size of 14 , and near-equivalent sample sizes for all species is recommended for such comparisons [48]. Conservatively, to account for any additional variation, we recommend a minimum sample size of 20 individuals per species for future studies.

\section{Discussion}

We confirmed that the neopterin molecule is detectable via commercial ELISA in the urine of three different platyrrhine families (Atelidae, Callitrichidae, and Pitheciidae) across six different genera. This is consistent with the use of this assay to test neopterin in other primate taxa [3, 36, 37, 39-44]. With nine biological validation studies across non-human primates that confirm neopterin is a nonspecific biological marker for cell-mediated immune function, this molecule can likely be used as a

Table 1 Summarized SG measurements of urine samples in this study. We sampled golden lion tamarins and white-faced sakis living in captivity, and wild emperor tamarins, saddleback tamarins, a brown titi monkey, and a Colombian red howler monkey. $n$ denotes number of samples analyzed from each species

\begin{tabular}{|c|c|c|c|c|c|c|}
\hline \multirow{2}{*}{$\begin{array}{l}\text { Sampling } \\
\text { location }\end{array}$} & \multirow[t]{2}{*}{ Species } & \multirow[t]{2}{*}{$n$} & \multicolumn{2}{|c|}{ SG at time of collection } & \multicolumn{2}{|c|}{ SG at time of analysis } \\
\hline & & & Mean & Stdev. & Mean & Stdev. \\
\hline \multirow[t]{2}{*}{ Captive } & Leontopithecus rosalia & 2 & 1.050 & 0.014 & 1.049 & 0.042 \\
\hline & Pithecia pithecia & 5 & 1.037 & 0.007 & 1.039 & 0.007 \\
\hline \multirow[t]{4}{*}{ Field } & Saguinus imperator & 46 & 1.012 & 0.006 & 1.011 & 0.005 \\
\hline & Leontocebus weddelli & 39 & 1.011 & 0.005 & 1.009 & 0.004 \\
\hline & Alouatta seniculus & 1 & $1.052^{\mathrm{a}}$ & - & $1.052^{\mathrm{a}}$ & - \\
\hline & Plecturocebus toppini & 1 & $1.014^{\mathrm{a}}$ & - & $1.014^{\mathrm{a}}$ & - \\
\hline
\end{tabular}


Table 2 Summarized urinary neopterin concentrations in this study. We sampled golden lion tamarins and white-faced sakis living in captivity, and wild living emperor tamarins, saddleback tamarins, a brown titi monkey, and a Colombian red howler monkey. All values are in $\mathrm{ng} / \mathrm{mL}$ corrected based on SG, as listed in the methods. $n$ denotes number of samples analyzed from each species

\begin{tabular}{lllll}
\hline Species & $n$ & Mean $(\mathrm{ng} / \mathrm{mL}$ corr. SG) & Median (ng/mL corr. SG) & Stdev. (ng/mL corr. SG) \\
\hline Leontopithecus rosalia & 2 & 1364.82 & 1364.82 & 99.31 \\
Pithecia pithecia & 5 & 392.55 & 339.38 & 269.96 \\
Saguinus imperator & 46 & 186.98 & 149.85 & 167.69 \\
Leontocebus weddelli & 39 & 478.25 & 300.29 & 375.98 \\
Alouatta seniculus & 1 & 2060.31 & - & - \\
Plecturocebus toppini & 1 & 1317.51 & - & - \\
\hline
\end{tabular}

non-invasive marker of immune system activation in platyrrhines, accompanied by prior validation experiments in untested species.

There was no significant change in measured SG between time of collection and time of analysis in these species, which suggests that the degradation of $\mathrm{Cr}$ can be inhibited by refrigeration. The variation in SG between the urine samples of captive and wild individuals could be attributed to species differences, although we hypothesize it is more likely due to diet. Unfortunately, sample sizes precluded the statistical testing of this hypothesis. Diet, however, can influence hydration and consequently the concentration of solutes in urine, both of which are reflected in the SG of urine samples [38]. However, the small sample size makes it difficult to confirm these are the only contributing factors to the SG variation observed in this study. Additionally, urine samples collected from captive $P$. pithecia turned from yellow to a black color when exposed to the air post collection, suggesting this individual had a rare inherited disease called alkaptonuria. Because the urine had turned black prior to performing the ELISA, it is possible that this impacted the colorimetric performance of the ELISA from P. pithecia; however, diluting the

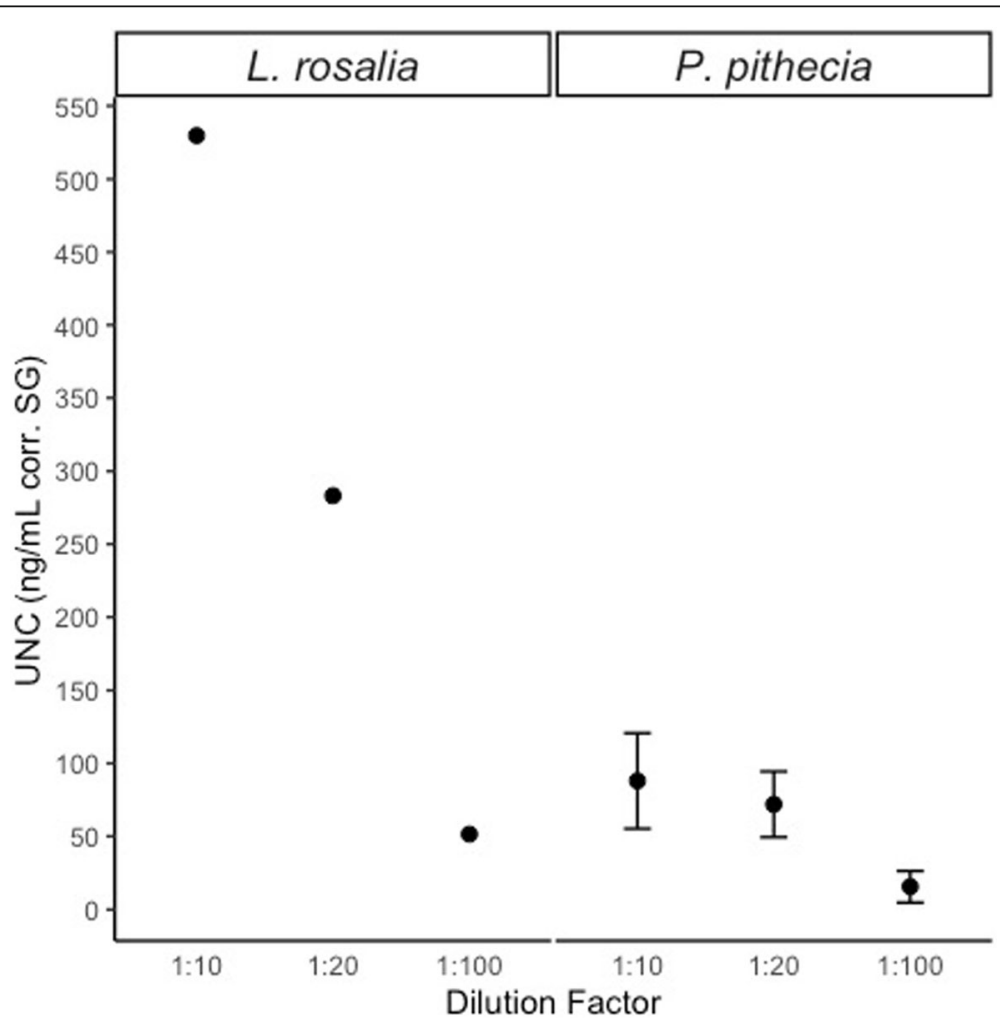

Fig. 1 Measures of UNC in samples collected from captive Leontopithecus rosalia $(n=1)$ and Pithecia pithecia $(n=5)$ diluted to three dilution factors. Samples were diluted in assay buffer and ran in duplicate. Data shown for $P$. pithecia is average \pm standard deviation. No error bars are shown for L. rosalia as there was only one sample included for this analysis 
samples 1:100 reduced the chance of interference in the assay.

Behringer et al. [39] found significant differences between the UNC of captive and wild chimpanzees, attributing this variation to increased immune challenges faced by wild chimpanzees [39]. Although we could not perform such comparisons with these data, we believe that comparing the immune status of captive and wild platyrrhine taxa would be valuable in the future. Longitudinal, repeated sampling from individuals across multiple seasons can facilitate the establishment of baseline UNC for populations to explore trends that have been observed in wild catarrhines, such as immunosenescence [36] and elevated UNC during disease outbreak [3]. Our power calculations indicate that minimum sample sizes per species from either the wild or captivity are $14(\beta=$ $0.8, \alpha=.05)$, a difficult standard to achieve across multiple genera due to the limited number of animals in captivity and the significant differences between captive conditions across these individuals.

The large inter-individual variation observed in S. imperator and $L$. weddelli exceeded measured intraindividual variation in individuals that were sampled multiple times. The non-significant intra-individual variation indicates that these preliminary UNC data are likely reliable measures of UNC on an individual basis and justifies our choice to use a single sample for these multiply sampled individuals in the rest of the analysis. We speculate that the differences in the UNC measured in samples from sympatric S. imperator and $L$. weddelli could be attributed to two factors: variation in speciesspecific baseline neopterin concentrations, varying degrees of parasite infection [49], or both. Inter-specific variation is expected as there is notable variation in published UNC values across catarrhine species [3, 36, 37, 39-44]. Within our study populations, $L$. weddelli has been observed to have higher overall parasite species richness and helminth prevalence compared to S. imperator [49]. More specifically, L. weddelli are parasitized by two filarial nematode species (Mansonella spp. and Dipetalonema spp), compared to $S$. imperator that are parasitized by just one (Dipetalonema spp.) [49]. Helminth infections have been shown to interact with Th1 immune mechanisms and elevate neopterin levels [50], which could explain the higher measured UNC in $L$. weddelli compared to S. imperator.

\section{Conclusion}

In conclusion, we measured the presence of urinary neopterin in platyrrhines, a non-invasive biomarker that has been confirmed to be associated with cell-mediated immune system activation in non-human primates [3, $36,37,39-44]$. Future studies are necessary to determine baseline UNC values across primate species to evaluate health trends and assess the adaptive response of UNC during the onset of disease and infection. Lahoz et al. [44] provides promising evidence that elevated neopterin is associated with increased immune system activity in one platyrrhine species. Their findings, coupled with our preliminary findings indicating higher measured UNC in $L$. weddelli, which have known higher blood parasite infection in these populations, suggests that UNC can be used as a measure to evaluate health status and disease outbreaks in wild and captive platyrrhine populations to predict survival outcomes in the future. It is likely that UNC in these species mirrors the same seasonal fluctuation of other non-human primates with similar time delays following infections, thus frequent sampling is recommended in future studies to adequately reflect the dynamic immune system response observed in humans and other non-human primates.

\section{Methods \\ Captive sample collection}

Seven urine samples were opportunistically collected from two golden lion tamarins ( $L$. rosalia) and two white-faced saki monkeys ( $P$. pithecia) in the Tropical Rainforest exhibit at the Woodland Park Zoo, Seattle, Washington from April 8 to 20, 2018. Five samples were collected from $P$. pithecia and two samples were collected from L. rosalia using swabs (Salivette. Cortisol, Sarstedt, Nürmbrecht, Germany) previously validated for non-invasive urine collection prior to UNC measurement [51]. The Salivette collection device has an upper compartment that houses a swab nested within a larger tube. This upper compartment has a hole at the bottom that allows for urine to be drawn out of the swab and pool within the tube when centrifuged. Using sterile technique, zookeepers removed the swab from the tube and swiped it over the pooled urine to collect and absorb the sample in its entirety. The zookeepers then used a manual oscillatory centrifuge composed of cardboard disks and string (a modified whirligig toy colloquially referred to as a "paperfuge") [52] to generate enough rotational force to recover the volume of urine absorbed in the swab. Zookeepers were not able to positively connect specific samples to individuals during captive sample collection. Urine samples were stored at $4-8^{\circ} \mathrm{C}$ in a refrigerator until analysis.

\section{Field sample collection}

The field study was performed at the Estacion Biologica Rio Los Amigos (EBLA) $\left(12^{\circ} 34^{\prime} 07^{\prime \prime} \mathrm{S}, 7^{\circ} 05^{\prime} 57^{\prime \prime} \mathrm{W}\right)$ in the Madre de Dios Department of southeastern Peru in collaboration with a longitudinal capture-and-release program by Field Projects International [53]. In brief, animals are habituated to a baited multi-compartment trap that they enter voluntarily. Entire groups are processed 
simultaneously and released upon recovery at the same trap site on the day of capture. Teams of five trained wildlife handlers process animals using a novel two-step anesthesia protocol [53]. Each individual is tagged permanently with a microchip (Home Again, Merck). During recovery from anesthesia, animals are placed in individual holding cages with urine collection trays beneath that are checked every three minutes. For this study, urine samples were opportunistically collected from 40 emperor tamarins (S. imperator) and 34 saddleback tamarins ( $L$. weddelli) during capture and release. We collected urine samples that were uncontaminated by soil, feces, or urine from other individuals. We collected and analyzed multiple samples from three saddleback tamarins and three emperor tamarins to assess intra-individual variation. Additionally, during primate follows, urine samples were opportunistically collected from one Colombian red howler monkey (A. seniculus) and one brown titi monkey (P. toppini) using Salivette collection devices (Salivette. Cortisol, Sarstedt, Nürmbrecht, Germany) to recover urine from the surface of leaves. All urine samples were stored immediately upon collection in portable cool packs at a temperature of $8^{\circ} \mathrm{C}$.

\section{Sample storage and specific gravity}

Upon return to the station, field samples were labeled in more detail and $10 \mu \mathrm{L}$ of urine were removed from each sample using a sterile pipette to determine the specific gravity using a handheld refractometer (Ade Optics TriScale Clinical Refractometer, Oregon City, USA) on the day of collection. Samples collected from captive animals by zookeepers also followed this protocol for measuring SG at time of collection. The remaining volumes of the recovered urine samples were then stored at room temperature sheltered from direct sunlight until further analysis. Urine samples from the field study were stored in a freezer at -5 to $-10^{\circ} \mathrm{C}$ until sample analysis.

\section{Urinary neopterin analyses}

Samples from captive individuals were analyzed at Central Washington University, whereas field sample analyses were performed at The Green Lab, a molecular field laboratory at Inkaterra Guides Field Station in Puerto Maldonado, Peru. To measure UNC, we used a commercially available Neopterin ELISA kit (Art. No. RE59321, IBL International GmbH, Hamburg, Germany) designed for quantifying neopterin in human serum plasma and urine. For captive sample analysis, three different dilution factors (1:10, 1:20, and 1:100) were used to confirm that the ELISA would identify neopterin in these species and to evaluate the efficacy and cross-reactivity of the kit. Previous work reported that a 1:100 dilution is adequate for non-human primates [36] and is also recommended by the manufacturer. Diluted samples were mixed by inverting the sample tube three to four times to fully homogenize the samples. All samples, standards, and controls were measured in duplicate when possible given plate size constraints (only two urine samples were measured once). The assay was performed using the manufacturer's instructions. Briefly, following dilution, $20 \mu \mathrm{L}$ of each sample, $100 \mu \mathrm{L}$ of enzyme conjugate, and $50 \mu \mathrm{L}$ antiserum were added to each well on the 96-well plate provided in the kit. The plate was then covered with black adhesive foil, wrapped in aluminum foil and incubated at room temperature for $90 \mathrm{~min}$. During this time, every $90 \mathrm{~s}$, the plate was gently manually shaken three times side-to-side and three times forward and backwards. Following the 90-min incubation period, the plate was washed four times using the provided wash buffer. Using a repeater pipette, $150 \mu \mathrm{L}$ of tetramethylbenzidine substrate solution was added into each well and incubated at room temperature for 10 min. The reaction was then stopped using $150 \mu \mathrm{L}$ stop solution. Although the manufacturer suggested that the optical density be measured at $450 \mathrm{~nm}$, due to the filter availability constraints while in the field, we decided to assess the efficacy of measuring the optical density above and below the recommended wavelength during captive samples analyses. To do so, the optical density was read photometrically at 405 , 450 , and $490 \mathrm{~nm}$ using a BioTek Synergy 2 (BioTek Instruments, Inc., Winooski, Vermont, USA). For field sample analysis, optical density was then read at 405 and $490 \mathrm{~nm}$ using a BioRad Model 550 Microplate Reader (BioRad Laboratories Inc., Hercules, California, USA). Inter-assay coefficients of variation, which were determined by repeated measurements of high and low value controls in each assay, were 4.39 and $3.19 \%$, respectively. A 4-Parameter Logistic standard curve was used to calculate the neopterin concentration of each sample.

To account for differences in urine volume and concentration, SG-corrected neopterin concentrations were calculated using the following formula (as per [54]):

$$
S G \text { corrected concentration }=\text { raw value } \times \frac{\left(S G_{\text {mean }}-1.0\right)}{\left(S G_{\text {sample }}-1.0\right)}
$$

The final SG value was used to control for variation in urine volume, density, and concentration. A decrease in SG is expected, corresponding to the degree of degradation of creatinine in the urine sample [37]. The final neopterin concentrations were transformed from $\mathrm{nmol} / \mathrm{L}$ to $\mathrm{ng} / \mathrm{mL}$ corr.SG following the manufacturer's protocol. 


\section{Statistical analyses}

Parametric tests were used for data analyses following a Gaussian distribution, verified by the Anderson-Darling test. Statistical tests were not possible when analyzing the results from the captive species due to their small sample size and the low power of null hypothesis tests [48]. Therefore, we used descriptive statistics to evaluate mean SG and UNC for each species population. Non-parametric Wilcoxon signed-rank tests were performed to investigate whether the SG of urine samples at the time of sample collection significantly differed from the SG of samples at the time of analysis. T-tests were used to compare UNC in samples collected from S. imperator and L. weddelli. Analyses were considered significant when $p<0.05$. All statistics were performed in R Studio 1.1.456.

\section{Abbreviations}

Cr: Creatinine; ELISA: Enzyme-linked immunosorbent assay; IFN-ү: Interferon gamma; SG: Specific gravity; Th1: T-helper 1; UNC: Urinary neopterin concentration

\section{Acknowledgments}

Logistical support was received in Peru from the Amazon Conservation Association and Field Projects International. We thank the Ministry of Agriculture for their support in conducting this research, and Inkaterra Guides Field Station for allowing us to use their facilities in the Green Lab. We would like to thank the following people for their involvement in the project: Ana Priscila Peralta Aguilar for her assistance working as a veterinarian on this project, field managers Gustav Steinhardt and Luke Fannin for their assistance with urine collection during primate follows, the Woodland Park Zoo primate keepers for their help with sample collection, and the Field Projects International research assistants who assisted with data and sample collection.

\section{Author's contributions \\ All authors have read and approved the manuscript. AS: conceptualization, methodology, funding acquisition, data collection, sample analysis, writing original draft. JAM: committee chair, conceptualization, supervision, draft review and editing, grant review and editing. MW: conceptualization, methodology, data collection, field logistics, supervision, draft review and editing. GE: data collection, field logistics, draft review and editing. AKB: methodology, laboratory supervision, sample analysis and interpretation of data, draft review and editing. \\ Funding \\ The acquisition of supplies and equipment for this research was financially supported by the Central Washington University (CWU) School of Graduate Studies and Research, the Pete and Sandra Barlow Award from the Department of Anthropology and Museum Studies, the Debra and Arlen Prentice Award for Primate Research from the Primate Behavior program, and Field Projects International.}

\section{Availability of data and materials}

The datasets used and analyzed during the current study are available from the corresponding author on reasonable request.

\section{Ethics approval and consent to participate}

All sampling protocols adhere to guidelines outlined by the American Society of Mammalogists [55] and were approved by authorities at the Woodland Park Zoo (WPZ\#2018-5) as well as the Institutional Animal Care and Use Committees at Central Washington University (A011802) and the University of Missouri-St. Louis (733363-5). The Peruvian Ministry of the Environment (SERFOR) granted annual research and collection permits. All sampling protocols complied with Ley No. 30407/2015 on Animal Welfare and Protection adopted by the Peruvian government. The Peruvian government does not require, nor does it provide a means for researchers to obtain ethics approval for wildlife research.

\section{Consent for publication}

Not applicable.

\section{Competing interests}

The authors declare that they have no competing interests.

\section{Author details}

${ }^{1}$ Department of Anthropology, Washington University in St. Louis, St. Louis, MO, USA. ${ }^{2}$ Primate Behavior Program, Central Washington University, Ellensburg, WA, USA. ${ }^{3}$ Department of Anthropology \& Museum Studies, Central Washington University, Ellensburg, WA, USA. ${ }^{4}$ Population

Sustainability, San Diego Zoo Institute for Conservation Research, Escondido, CA, USA. ${ }^{5}$ Department of Biology, University of Missouri-St. Louis, St. Louis, MO, USA. ${ }^{6}$ Field Projects International, St. Louis, MO, USA. ${ }^{7}$ Washington University School of Medicine in St. Louis, St. Louis, MO, USA. ${ }^{8}$ Department of Biological Sciences, Central Washington University, Ellensburg, WA, USA.

Received: 19 July 2019 Accepted: 6 February 2020

Published online: 27 February 2020

\section{References}

1. Karesh WB, Cook RA. Applications of veterinary medicine to in situ conservation efforts. Oryx.1. Karesh WB, Cook RA. Applications of veterinary medicine to in situ conservation efforts. Oryx. 1995;29(4): 244-52.

2. Deem SL, Karesh WB, Weisman W. Putting theory into practice: wildlife health in conservation. Conserv Biol. 2008;15(5):1224-33.

3. Wu DF, Behringer V, Wittig RM, Leendertz FH, Deschner T. Urinary neopterin levels increase and predict survival during a respiratory outbreak in wild chimpanzees (Taï National Park, Côte d'Ivoire). Sci Rep [Internet]. 2018;8(1): 13346 Available from: http://www.nature.com/articles/s41598-018-31563-7.

4. Vié JC, Moreau B, de Thoisy B, Fournier P, Genty C. Hematology and serum biochemistry values of free-ranging red howler monkeys (Alouatta seniculus) from French Guiana. J zoo Wildl med [internet]. 1998;29(2):142149. Available from: http://www.ncbi.nlm.nih.gov/pubmed/9732027.

5. Crissey SD, Silva JCS, Meehan T, Slifka KA, Bowen PE, Stacewicz-Sapuntzakis $\mathrm{M}$, et al. Nutritional status of free-ranging Mexican howler monkeys (Alouatta palliata mexicana) in Veracruz, Mexico: serum chemistry; lipoprotein profile; vitamins D, a, and E; carotenoids; and minerals. Zoo Biol. 2003;22(3):239-51.

6. Junge RE, Louis EE. Biomedical evaluation of black lemurs (Eulemur macaco macaco) in Lokobe reserve, Madagascar J Zoo Wildl Med 2007;38(1):67-76.

7. Junge RE, Louis EE. Preliminary biomedical evaluation of wild ruffed lemurs (Varecia variegata and V. rubra). Am J Primatol. 2005;66(1):85-94.

8. Soto-Calderón ID, Acevedo-Garcés YA, Álvarez-Cardona J, Hernández-Castro C, García-Montoya GM. Physiological and parasitological implications of living in a city: the case of the white-footed tamarin (Saguinus leucopus). Am J Primatol. 2016;78(12):1272-81.

9. Fox M, Brieva C, Moreno C, Macwilliams P, Fox M, Brieva C, et al. Hematologic and serum biochemistry reference values in wild-caught white-footed tamarins (Saguinus leucopus) housed in captivity. J Zoo Wildl Med. 2008;39(4):548-57.

10. Crofoot MC, Norton TM, Lessnau RG, Viner TC, Chen TC, Mazzaro LM, et al. Field anesthesia and health assessment of free-ranging Cebus capucinus in Panama. Int J Primatol. 2009:30(1):125-41.

11. Teixeira MG, Ferreira AF, Colaço AA, Ferreira SF, de Melo Benvenutti ME, Queiroga FLPG. Hematologic and blood chemistry values of healthy Cebus flavius kept in northeast of Brazil. J Med Primatol. 2013;42(2):51-6.

12. Silva I de $O$ e, Silva F de FR da, Fuzessy LF, Tavela A de O, Júnior MC, Silva VHD E, et al. hematology and blood biochemistry in wild hybrid marmosets from the Atlantic Forest, Brazil. Ciência Rural 2014;44(9):1596-1602.

13. García-Feria LM, Chapman CA, Pastor-Nieto R, Serio-Silva JC. Biochemical and hematological evaluations of black howler monkeys (Alouatta pigra) in highly degraded landscapes in Mexico. J Med Primatol. 2017;46(6):304-10.

14. Beehner JC, Mccann C. Seasonal and altitudinal effects on glucocorticoid metabolites in a wild primate (Theropithecus gelada). Physiol Behav. 2008; 95(3):508-14.

15. Setchell JM, Smith T, Wickings EJ, Knapp LA. Stress, social behaviour, and secondary sexual traits in a male primate. Horm Behav. 2010;58(5):720-8.

16. Shutt K, Setchell JM, Heistermann M. Non-invasive monitoring of physiological stress in the Western lowland gorilla (Gorilla gorilla gorilla): 
validation of a fecal glucocorticoid assay and methods for practical application in the field. Gen Comp Endocrinol. 2012;179(2):167-77.

17. Foerster S, Cords M, Monfort SL. Seasonal Energetic Stress in a Tropical Forest Primate: Proximate Causes and Evolutionary Implications. PLoS ONE. 2012;7(11):e50108

18. Murray CM, Heintz MR, Lonsdorf EV, Parr LA, Santymire RM. Validation of a field technique and characterization of fecal glucocorticoid metabolite analysis in wild chimpanzees (pan troglodytes). Am J Primatol. 2012;75(1): 57-64.

19. Barelli C, Albanese D, Donati C, Pindo M, Dallago C, Rovero F, et al. Habitat fragmentation is associated to gut microbiota diversity of an endangered primate: implications for conservation. Scientific Reports. 2015;5(1):14862.

20. Gomez A, Petrzelkova K, Yeoman CJ, Vlckova K, Mrázek J, Koppova I, et al. Gut microbiome composition and metabolomic profiles of wild western lowland gorillas (Gorilla gorilla gorilla) reflect host ecology. Mol Ecol. 2015; 24(10):2551-65.

21. Amato KR, Metcalf JL, Song SJ, Hale VL, Clayton J, Ackermann G, et al. Using the gut microbiota as a novel tool for examining colobine primate $\mathrm{Gl}$ health. Global Ecology and Conservation. 2016;7:225-37.

22. Amato KR, Yeoman CJ, Kent A, Righini N, Carbonero F, Estrada A, et al. Habitat degradation impacts black howler monkey (Alouatta pigra) gastrointestinal microbiomes. The ISME Journal. 2013;7(7):1344-53.

23. Nagpal R, Shively CA, Appt SA, Register TC, Michalson KT, Vitolins MZ, et al. Gut microbiome composition in non-human primates consuming a western or Mediterranean diet. Front Nutr. 2018:5:28.

24. Trosvik P, Rueness EK, De Muinck EJ, Moges A, Mekonnen A. Ecological plasticity in the gastrointestinal microbiomes of Ethiopian Chlorocebus monkeys. Sci Rep. 2018;8(1):1-0.

25. Trosvik P, de Muinck EJ, Rueness EK, Fashing PJ, Beierschmitt EC, Callingham $\mathrm{KR}$, et al. Multilevel social structure and diet shape the gut microbiota of the gelada monkey, the only grazing primate. Microbiome. 2018;5.

26. Deschner T, Kratzsch J, Hohmann G. Urinary C-peptide as a method for monitoring body mass changes in captive bonobos (pan paniscus). Horm Behav. 2008;54(5):620-6.

27. Thompson ME, Knott CD. Urinary C-peptide of insulin as a non-invasive marker of energy balance in wild orangutans. Horm Behav. 2008;53(4):526-35.

28. Higham JP, Girard-Buttoz C, Engelhardt A, Heistermann M. Urinary C-peptide of insulin as a non-invasive marker of nutritional status: Some practicalities. PLoS ONE. 2011;6(7):e22398.

29. Girard-Buttoz C, Higham JP, Heistermann M, Wedegärtner S, Maestripieri D, Engelhardt A. Urinary C-peptide measurement as a marker of nutritional status in macaques. PLoS One. 2011;6(3):e18042.

30. Huber C. Immune response-associated production of neopterin. Release from macrophages primarily under control of interferon-gamma. J Exp Med. 1984;160(1):310-6.

31. Murr C, Widner B, Wirleitner B, Fuchs D. Neopterin as a marker for immune system activation. Curr Drug Metab. 2002;3(2):175-87.

32. Fuchs D, Hausen A, Reibnegger G, Werner ER, Dierich MP, Wachter H. Neopterin as a marker for activated cell-mediated immunity: application in HIV infection. Immunol Today. 1988;9(5):150-5.

33. Abita JP, Cost H, Milstien S, Kaufman S, Saimot G. Urinary neopterin and biopterin levels in patients with AIDS and AIDS-related complex. Lancet. 1985:326(8445):51-2.

34. Frick B, Schroecksnadel K, Neurauter G, Leblhuber F, Fuchs D. Increasing production of homocysteine and neopterin and degradation of tryptophan with older age. Clin Biochem. 2004;37(8):684-7.

35. Hawkley LC, Cacioppo JT. Stress and the aging immune system. Brain Behav Immun. 2004;18(2):114-9.

36. Müller N, Heistermann M, Strube C, Schülke O, Ostner J. Age, but not anthelmintic treatment, is associated with urinary neopterin levels in semifree ranging Barbary macaques. Sci Rep, 2017;7(1):1.

37. Heistermann M, Higham JP. Urinary neopterin, a non-invasive marker of mammalian cellular immune activation, is highly stable under field conditions. Sci Rep [Internet]. 2015:5(1):16308 Available from: http://www. nature.com/articles/srep16308.

38. Anestis SF, Breakey AA, Beuerlein MM, Bribiescas RG. Specific gravity as an alternative to creatinine for estimating urine concentration in captive and wild chimpanzee (pan troglodytes) samples. Am J Primatol. 2009;71(2):130-5.

39. Behringer $V$, Stevens JMG, Wittig RM, Crockford C, Zuberbuhler K, Leendertz $\mathrm{FH}$, Deschner T. Elevated neopterin levels in wild, healthy chimpanzees indicate constant investment in unspecific immune system. BMC Zoology. 2019:4:2.

40. Behringer V, Stevens JMG, Leendertz FH, Hohmann G, Deschner T. Validation of a method for the assessment of urinary neopterin levels to monitor health status in non-human-primate species. Front Physiol. 2017; 8(FEB):1-11.

41. Fendrich C, Lüke W, Stahl-Hennig C, Herchenröder O, Fuchs D, Wachter H, et al. Urinary neopterin concentrations in rhesus monkeys after infection with simian immunodeficiency virus (SIVmac 251). 1989;3(5):305-8.

42. Higham JP, Kraus C, Stahl-Hennig C, Engelhardt A, Fuchs D, Heistermann M. Evaluating noninvasive markers of nonhuman primate immune activation and inflammation. Am J Phys Anthropol. 2015;158(4):673-84.

43. Löhrich T, Behringer V, Wittig RM, Deschner T, Leendertz FH. The use of neopterin as a noninvasive marker in monitoring diseases in wild chimpanzees. Ecohealth. 2018:15(4):792-803.

44. Lahoz M, Kauffman MA, Carfagnini J, Vidal A, Papouchado M, Sterin-Prync A, et al. Pharmacokinetics and pharmacodynamics of interferon beta 1a in Cebus apella. J Med Primatol. 2009;38(3):187-91.

45. Seino S, Steiner D, Bell G. Sequence of a New World primate insulin having low biological potency and immunoreactivity. Proc National Acad Sci. 1987; 84(21):7423-7

46. Alfaro JWL. Special issue: phylogeny and biogeography of Neotropical primates. Mol Phylogenet Evol. 2015;82:345-7.

47. Seminoff JA. The IUCN red list of threatened species - version. 2014:3:2004

48. Cohen J. Statistical power analysis for the behavioral sciences; 1988

49. Erkenswick GA, Watsa M, Gozalo AS, Dudaie S, Bailey L, Muranda KS, et al. A multiyear survey of helminths from wild saddleback (Leontocebus weddelli) and emperor (Saguinus imperator) tamarins. Am J Primatol. 2019;81(12): e23063.

50. Zander R, Butler N. Dysfunctional Adaptive Immunity During Parasitic Infections. Curr Immunol Rev. 2014:9(3):179-89.

51. Danish LM, Heistermann M, Agil M, Engelhardt A. Validation of a novel collection device for non-invasive urine sampling from free-ranging animals. PLoS ONE. 2015;10(11):e0142051.

52. Bhamla MS, Benson B, Chai C, Katsikis G, Johri A, Prakash M. Hand-powered ultralow-cost paper centrifuge. Nature Biomedical Engineering. 2017;1(1):0009.

53. Watsa M, Erkenswick G, Halloran D, Kane EK, Poirier A, Klonoski K, et al. A field protocol for the capture and release of Callitrichids. Neotrop Primates. 2015;22(2):59-68.

54. Miller RC, Brindle E, Holman DJ, Shofer J, Klein NA, Soules MR, et al. Comparison of specific gravity and creatinine for normalizing urinary reproductive hormone concentrations. Clin Chem. 2004;50(5):924-32.

55. Sikes RS. 2016 guidelines of the American Society of Mammalogists for the use of wild mammals in research and education. J Mammal. 2016; 97(3):663-88

\section{Publisher's Note}

Springer Nature remains neutral with regard to jurisdictional claims in published maps and institutional affiliations.

\section{Ready to submit your research? Choose BMC and benefit from:}

- fast, convenient online submission

- thorough peer review by experienced researchers in your field

- rapid publication on acceptance

- support for research data, including large and complex data types

- gold Open Access which fosters wider collaboration and increased citations

- maximum visibility for your research: over $100 \mathrm{M}$ website views per year

At BMC, research is always in progress.

Learn more biomedcentral.com/submissions 